\title{
ROADMAP TO SUCCESS: YOUR FIRST SIMULATION MODEL
}

\author{
Robin Clark \\ The QMT Group \\ 1143 Oak Ridge Turnpike, Suite 134 \\ Oak Ridge, TN 37830, USA \\ David Krahl \\ Imagine That Inc \\ 6830 Via Del Oro, Suite 230 \\ San Jose, CA 95119, USA
}

\begin{abstract}
Success in your first simulation model is crucial for the overall prospects of a new simulation program. Here we will outline the building of your first simulation model with emphasis on the overall process and what it takes to be a successful model builder.
\end{abstract}

\section{INTRODUCTION}

Building a simulation model is like a road trip. You have to decide on your destination, choose your companions, plan the route, select an appropriate vehicle, record the events of the journey, and report home when the trip is done. It has also been said that it's not a real road trip unless there was at least one Uturn.

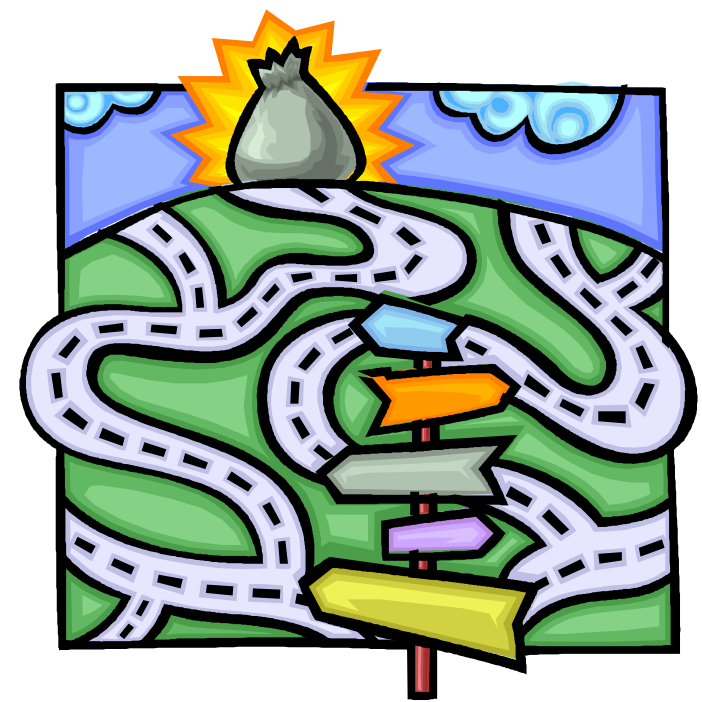

Simulation software has come a long way since its inception. For the most part, a simulation modeler now works with a modern simulation environment that includes many tools for model construction and analysis. However, there are still many challenges that face a first-time modeler. Depending on your background and the type and scale of the system, you may need to acquire skills for model abstraction, project management, and model building. Perhaps some programming skills will also need to be learned or remembered from a college class. A first simulation model can be successful if planned well. This requires reasonable expectations, time to dedicate to the modeling process, a willingness to learn, and support from management. 


\section{Clark, and Krahl}

This roadmap, if followed, will increase the probability that your first simulation project will be successful. Even if you have already completed a project or two, this advice may help with future models. The authors have helped hundreds of modelers get started. The information here is based on that experience and the observations of successful simulationists.

\section{FINDING A PROJECT}

Where are we going on our trip? It's probably wise not to embark on a cross-country trip with a newly acquired driver's license. A new driver should choose a trip that can be easily completed in a single day, avoids complex traffic situations, and takes advantage of good weather.

The first simulation project should be one that is likely to be successful. In this case, success is defined as a simulation model that demonstrates value to the organization within a relatively short time frame. The ideal process to model for the first project will be complex enough so that the behavior will not be entirely obvious, yet simple enough to be modeled straightforwardly. Often a small model can be very useful in identifying key information about a process. Don't begin by modeling your entire supply chain. Instead, model a single, interesting and important inventory point.

Another property of a good starting project is management support. If you have the luxury of choosing between different departments, select the one with the more "enlightened" manager. Their support is important for a variety of reasons which will be covered in the Team section of this paper.

\section{MODEL SCOPE}

For your first road trip, what is your objective? Are you prepared for bad weather? How far can you realistically go in a day? A little planning will go a long way towards your enjoyment of the trip.

After selecting the appropriate first project, the model scope must be developed. The model scope includes the primary questions the model will be developed to answer and the key performance measures needed to answer the questions. Often there are several related questions such as "do I need an additional machine" and "how should the new machine be staffed". These questions are critical in determining the level of detail needed in the model. Musselman (1993) states "Don't look for a solution until first listening to the problem". Too many models are built without clear objectives in mind; this behavior leads to overly complex models that often answer the wrong question.

The model scope also contains well-defined process boundaries. This can be easily illustrated by a high-level process flow chart depicting where the system starts and stops. This will determine which processes are included in the study. Without the process boundaries, the scope of the project can easily expand, which can be disastrous if not managed.

Do not include questions in the model scope that could make you lose sight of the main focus of the simulation project. Questions regarding details that lie outside the identified process flow are usually easily recognized. However, a question that is indeed related to the processes being modeled but is too detailed for the current model scope can be difficult to identify for a new modeler. Be aware that the additional level of detail will delay answering the questions related to the primary focus of the project. If you are not sure about a the importance of a particular facet of the system, consider building a small model to study the impact of that factor.

Of course, expect that additional needs and questions will arise during the project. When these come up, carefully consider whether or not the model scope should be re-evaluated. Both the difficulty in introducing any changes to scope as well as the significance of the changes to the final result should be weighed. For example, a big change that is not anticipated to have a significant impact on model results should not be included. It also may be possible to develop a prototype model to evaluate if the proposed changes will significantly alter the model.

A U-turn or two should be expected in this phase of the modeling process. If the model scope gets changed significantly, that could be a sign you have troubling issues. It is recommended that you stay the 


\section{Clark, and Krahl}

course once you nail down the model scope and leave new questions that require significant model enhancements until after the first project has been completed.

\subsection{Assumptions Document}

The assumptions document should be started at the beginning of the project. This document includes the assumptions made in the model in order to make the model building process easier. It should be a living document that gets updated throughout the modeling process. This document is also useful when the model gets reused later.

\subsection{Process Documentation}

The process document describes the process flow in detail. It is the basis by which the modeler will decide how to conceptualize the model. The detail included will help to determine the appropriate model assumptions, avoiding modeling errors or rework. This document includes:

- Logic of how the entities interact with the real system

- Detailed flow charts of the system.

- A description of how the resources are used.

This document is used to clarify how the system actually works. Since it describes the real-world system, it should not change throughout the model building process unless the actual process or your understanding of the process changes. This document should be validated with subject matter experts before proceeding to any other step.

The modeler will use this information to develop an understanding of the behavior of the process. The amount of detail depends on the modeler's experience with the system. If the modeler typically works with this process on a day-to-day basis, less detail is necessary than if the process documentation is used to communicate to an outside consultant.

\section{THE TEAM}

Imagine travelling with a group of dissimilar people who must all work together to meet a common goal. The ultimate road trips, exploring expeditions such as Shackleton's adventure near the south pole, have been made or broken on the quality of the team and its leadership. As the leader of the expedition, you have the most important position. You will be leading the group into uncharted territory.

A simulation project requires several team members, not just one person. Each person should bring unique skills and information to the project. At a minimum, the team needs to include the model builder, a management champion, and subject matter experts.

\subsection{All Team Members}

Beyond technical expertise, management ability, and system knowledge, there are two important qualities to look for in a team member. The first quality is an excitement for simulation. This enthusiasm can overcome many obstacles and shortcomings. The next quality is availability. An appropriate amount of time needs to be set aside for the project. As with any type of project, including people on the team who are either not interested in the project or really do not have the time does more harm than good.

\subsection{The Modeler}

A good simulation modeler will have a broad range of skills. Most obviously, the modeler must be able to understand the statistics and random nature of most simulation models. Because the vast majority of simulation models include random behavior, the modeler will be required to understand some basic statistical concepts. There must be a comfort level with terms such as mean, standard deviation, replication, and confidence interval. 


\section{Clark, and Krahl}

Another attribute of a good modeler is an exposure to computer programming. In creating any simulation model, whether it entails writing code, connecting blocks together, or even filling out tables, the model builder is giving a set of instructions to the computer. A knowledge of basic programming concepts will help the modeler to better relate to how the computer interprets the model. An analogy here is that every pilot should understand something about aerodynamics.

The final, and in the authors' view the most important, attribute is common sense. The modeler needs to recognize a number of situations such as when:

- The model is generating implausible results

- A model is providing new information that is not intuitive

- A model is sufficiently complex that it is beyond the skills of the modeler

- Deadlines and expectations of the model by others are not realistic

- The level of model detail is not appropriate

Absent this savvy, the modeler can blunder into a project and/or create a model that will not produce the desired results in the required timeframe. A failure on the first model may doom any future models to the scrap heap.

The model builder should also be fairly proficient in the simulation tool. If this is the first simulation project for the model builder, training might be necessary to get the model builder up to speed in a timely matter. For a very simple model it might not be necessary. However if time is a critical factor and the system is complex, then training would be necessary for a new model builder.

\subsection{The Subject Matter Experts}

The subject matter experts (SME) are necessary because no single person fully understands the entire system. Even if a subject matter expert were familiar with the entire system, the model should be based on more than a single person's perspective. The SME should have detailed knowledge about the details of the operation. Not only how it should operate, but how it actually does operate, as there can often be a significant difference between these two. SME's can be brought into the modeling process at different stages. A production engineer might be brought into the modeling process at the beginning while an operator would later provide details on the behavior of a specific step in the process. SME's can be found in unlikely places. Don't be afraid to get the opinion of anyone who is involved with the system.

\subsection{The Management Champion}

The management champion is necessary in order to give the team the resources needed to accomplish the task at hand. The most important of these are allocating time for the model developer to build the model and influence to obtain simulation data. Other resources include access to key decision makers, the required hardware, and software, and a definition of the overall simulation inputs and expected results. The champion should also defend and manage simulation model expectations to other areas of the organization. A simulation champion can reside at any one of a number of levels in the company, from project manager to VP or even " $\mathrm{C}$ " level. The exact role of the champion is somewhat defined by their level within the organization.

\subsection{Other Team Members}

Outside resources, such as consultants, can be useful and should be carefully considered as well. Consultants could be used to completely build the model, just assist when difficulties arise, or to mentor the new model builder through their first couple of projects. Including a consultant on the team can keep a new model builder on the right path and can save a tremendous amount of time. It can also help ensure a successful first project. If the first project is not successful then chances are low that simulation takes root at that organization. The desirability of using an outside consultant depends on a number of factors including the complexity of the model, the background of the modeler, and the project timeline and budget. 


\section{Clark, and Krahl}

\section{DOCUMENTATION}

While it's not always necessary to document every phase of your road trip, there are benefits to recording the adventure. In this case, you are scouting for future travels. You may want to remember if the route worked out well, communicate to others about where to go (and not to go), report on your trip when you get home, and you may want to post the highlights to Facebook as you travel.

Documentation is critical to every phase of the simulation project. With the typical tight time schedule of a simulation project, this is an easy step to scrimp on, but, like balancing a checkbook, proper documentation will actually save time and energy. Proper documentation must be done to keep the project focused on the right problem, reduce the amount of model rework, and provide a record of the project status, assumptions, and objectives. The documentation that should be done at each phase of the project is described below:

- Assumptions: describes the decisions made about the modeling of the process. Which details are included and omitted including the overall level of modeling detail.

- Model scope: describes the beginning and ending points of the model as well as the purpose of the model.

- Process: provides the details of the operation of the real-world system.

- Conceptual: how the real-world system has been abstracted into the simulation model.

- Data: a discussion of the data structures and content, and how the modeler or end user can modify the data to exercise the model under different conditions.

- User documentation: instructs an end-user (non-modeler) on how to utilize the model. This may include elements of the data document oriented toward the person running the model.

- Developer documentation: the nuts and bolts of how the model works, focusing on the major and more complicated elements of the model. Often, this can be done most effectively within the model itself.

- Final report: includes the analysis, recommendations, and any information useful in making decisions based on the model.

\section{DATA}

Do you have all of the information necessary to make your trip? In the old days, we would go to the autoclub to pick up maps and get advice about the attractions on the way. This kind of planning and data collection will make the trip more enjoyable for all.

Getting all of the information required to build a simulation model is always the bottleneck of the entire process. Begin the process of documenting and collecting data as soon as possible. This should be done before the modeling process begins, typically while developing the process documentation and the model conceptualization.

Data can come from many places such as data collection systems, time studies, interviews of subject matter experts, and historical records. Identify the data sources and the people that control that data as part of the data collection process.

Before spending time gathering data on the system, spend some time analyzing exactly what type of data is needed. For example, if you are building a model of an emergency department to study the provider staffing, then you must know the amount of time the providers spend with the patients. Knowing the time the patients occupy the room would not help in the staffing model because the data includes both waiting time as well as service time. However, if you are building a model of the same emergency department to study the number of rooms needed, then knowing the time the patients spent in the room is useful. So, documenting the data needed before the starting the collection process can eliminate the energy wasted on collecting the wrong data.

The data documentation describes both the input tables and the output tables. For each table, the data documentation thoroughly describes the field structure and the record structure. The actual data is not needed before the model building stage but a clear idea of the data structure is necessary. Definitions 


\section{Clark, and Krahl}

should be clearly specified. Don't assume everyone will interpret the definition of parameters and results the same as you. A perfect example of this would be the calculation of utilization. Everyone understands that utilization is a measure of how much a resource is used, but there are many variations of how it is calculated.

The data documentation should also describe if the model could handle a structure change to the table. For example, what would the model do if the user added more rows to an input table? Would the model be able to automatically use the new data? Because of adding new rows to one table, would the user also have to add the same rows to other tables that have the same structure? What would the model do if the user deleted rows to an input table?

The data documentation should also describe the output tables. It is necessary to identify the results that should be collected as output. Take note that collecting too much output data can confuse and overwhelm the user, thus should be avoided.

\section{CONCEPTUALIZATION}

There are many ways to take a road trip. Perhaps you want to bicycle across Europe, or take a train along the coast. Perhaps your trip involves flying to a location and then driving from there. You need to choose the vehicle that meets your needs, abilities, and time frame.

A real world process must be abstracted into a conceptual model. It is not possible to completely know the behavior of the real system and it is impractical to incorporate every detail no matter how insignificant. A general rule of thumb is to build the simplest model that solves the problem. This is one of the reasons why it is important to focus on the problem statement throughout the model building process. There are many reasons why a simpler model is better. They take less time to develop, run, and analyze. They tend to be more reliable and flexible. And a smaller, less complex model is easier to explain and transfer to others (Kotiadis and Robinson 2008).

For some models the process of abstracting the real system into the simulation model is quite straightforward. For other systems, there can be multiple conceptual models each with its own set of advantages and disadvantages. For example, most simulation software tools contain the ability to model a conveyor. If the conveyor in the real system is used only to transport material, then it is generally best to map the conveyor element in the simulation software to the real-world conveyor. However, if the conveyor in the real system performs a number of other functions, it may be better to use different modeling constructs to simulate that conveyor. This may require more work on the part of the modeler, but it may be required to create an accurate representation of the actual process.

\subsection{Conceptualization Styles}

Models may be conceptualized to literally represent the system or be more logical in nature. A literally oriented model will have a more-or-less one to one relationship between the model elements and the elements in the real system. If the real system has 20 waiting areas for patients, the literal model will have the same number of queues. A logically oriented model will be more abstract, generally representing multiple elements in the real system with a single set of simulation components. The 20 queues may be represented by a single logical queue, perhaps with some additional logic to differentiate the literal queues.

Table 1: Model Conceptualization Styles

\begin{tabular}{|l|l|}
\hline \multicolumn{1}{|c|}{ Literal model } & \multicolumn{1}{c|}{ Logical model } \\
\hline Easy animation & Very scalable \\
\hline Easier to explain to others & Faster execution time \\
\hline Straightforward conceptualization & Smaller model size \\
\hline
\end{tabular}




\section{Clark, and Krahl}

Each of these approaches has its own advantages and disadvantages as illustrated in Table 1. It's probably best to begin with the literal modeling style and transition into logical models as your modeling skills improve.

Generally, a good place to start with model conceptualization is with the data. This is particularly true of systems with large or complex data requirements such as a supply chain. Begin by mapping the data sets and their relationships to each other. This serves a number of purposes:

- The data requirements can be easily communicated to everyone involved in the simulation project

- A well defined model database will assist with the structuring of the simulation model

- Any missing data will be exposed early in the modeling process

Note that this process does not require complete data, it defines what the structure of the data will look like. Once the data has been defined, the modeler can begin building the logic of the model. Start small and add detail. This allows for stepwise validation and makes for easier debugging.

\subsection{Conceptualization Documentation}

The Model Conceptualization document describes the approach the modeler will take to build the model. This document will describe why the modeler will build it that way. This could be due to model run time, model animation, scalability, or even due to the user interaction with the model.

\section{MODEL CONSTRUCTION}

Hopefully, your road trip will be enjoyable, with many new sights and experiences. It is said that "A journey of a thousand miles begins with a single step". Take your trip in small, manageable pieces.

Simulation model construction is typically an iterative process. The most important piece of advice for a model builder, whether it is your first or hundredth model, is to start small, verify and validate, add more, verify and validate, and so on. By using this iterative modeling process, you can evaluate the impact of each change and easily correct any modeling errors. If you were to build even a medium size model all at once, the validation and verification process could be daunting. Also, by building a progressively larger model, management and subject matter experts will have the opportunity to weigh in on the model as it is being constructed.

Don't forget to focus on the problem at hand. It is easy to get caught up in the minutia of the process which will not have any effect on the model results. If the model building is getting cumbersome, take a step back and ask some questions:

- Are there any simplifying assumptions?

- Is there a better way to conceptualize the model?

- Am I adding too much detail?

If possible, try to separate the model logic from the data. This will make it easier to build the model without complete data and to refine the data at a later time. For example, instead of directly entering a delay in a critical activity, provide a reference to a delay time that is stored in a spreadsheet or database. This may take a little extra time to do, but it will save a significant amount of time over the life of the model.

The process of creating the model is often as useful as the analytical results. Abstracting a real system into a simulation model will reveal details that are not readily apparent. For example, a simulation of an automotive production line revealed a simple timing issue that was a significant bottleneck to the overall manufacturing process. The "back-of-the-envelope" calculation that was done for the simulation model revealed this problem earlier than it would have been otherwise. You should also be ready to react to new information that arises from model development. This may change the detail of the model, the data required, or even the need for the simulation model itself. 


\subsection{Verification and Validation}

Verification and validation are important steps in the model building process. Verification is ensuring that the model is behaving as the modeler expects it to. Validation means that the model is a reasonable representation of the real system. Entire papers and book chapters are dedicated to this topic, so it is recommended that you consult one of the many good resources on the details of model verification and validation including (Banks et al. 2010), (Sargent 2009) and (Law 2007). Remember that a model is validated and verified for a specific purpose. If the questions asked of the model change, then verification and validation must be performed again.

\subsection{Debugging}

A single block in a modern simulation program may contain thousands of lines of code. The modeler assumes that this code behaves in an expected manner. Unfortunately, the developer or a different modeler may have a different expectation. In many cases, there may be many technically correct behaviors. It is important that the modeler understand how the model is working. A description of simulation debugging techniques and issues can be found in (Krahl 2005).

\subsection{Know When to Quit}

Simulation modeling can be fun. As the model nears completion, resist the urge to continue to add details and features that do not significantly enhance the utility of the model. If there is a little extra time at the end of the project, spend it documenting, testing the model, and performing some sensitivity analysis.

\subsection{Model Documentation for the User}

The model documentation for the user describes to the how the user will interact with the model. It should contain how to modify the model inputs, run the model, understand the animation, and interpret the results. The document should describe all the things a user should be allowed to do with the model. This documentation could be and often is, included in the model itself.

It is necessary to either provide the user with Model Scope, Assumptions Document, Process Document, and Data Document or include the pertinent items from each into this document.

\subsection{Model Documentation for the Developer}

The model documentation for the developer describes to a model developer how the model works. It should describe how each construct works, the calculations being made, and the logic being used. This documentation could be, and often is, included in the model itself. This documentation should be developed as the model is being built. It will be used later on when the model needs to be modified or reused.

\section{RESULTS}

Remember that your first simulation model is essentially a scouting trip. You will want to report back what your experiences were, whether the destination was worthwhile, and perhaps any new insights that you gained during your trip.

After the model has been built, verified, debugged, and validated, it is time to conduct the experiments on the model that was originally envisioned in the model scope phase. The goal should be to gain a fundamental understanding of the system, enough to know what the true system drivers are. Look for reports, graphs, and tools that help you to understand the system and the interactions between the components. If an inventory level is cyclical, it will be better to evaluate a plot of inventory over time rather than a single average inventory value. 


\subsection{Analytical Results}

Since most simulation models incorporate random behavior, all dedicated simulation tools will report the average values for a single run or period of time as well as statistics over multiple runs and time periods. If your model has any randomness, it is important that you do calculate some basic statistics over multiple runs or time periods. You should take full advantage of the tools provided by your simulation software for this analysis. Simulation textbooks such as (Law 2007) and (Banks et al. 2010) have a full description of the theory and examples of this type of analysis.

\subsection{Plots and Charts}

Graphical data representations are very useful ways to communicate simulation results to a range of people. Many simulation oriented graphs show not only the mean value, but a measure of the variance of the results. Time series plots allow you to easily see the cyclical nature of the system, can give an indication of how the system reacts to critical events such as a machine breakdown, and can even indicate whether or not the model has been run long enough to produce accurate steady-state results. Pie and bar charts can be used to illustrate the system performance to all members of the team regardless of their analytical background.

\subsection{Animation}

Model animation can be used to illustrate process interactions and system behavior. This is not a substitute for more rigorous analysis, but you can use this as a powerful tool for convincing others of the validity of your analytical results.

Animation can take on a variety of forms. These range from 2D automatic animations that show the actions of the model to 3D animations that show a visually realistic representation of the real system. Ironically, a more realistic animation can be detrimental as the audience will sometimes focus on inconsequential details ("our forklifts are blue, not yellow") rather than the overall system performance.

\subsection{Design of Experiments}

Using a design of experiments (DOE) approach to running the model and analyzing results can give you a more complete picture of model behavior in fewer runs. DOE tools can identify the most significant factors in model performance and provide sensitivity analysis features.

\subsection{Final Presentation}

At the end of a successful project, the team should sell the success to others in the organization. It was a big commitment for the entire team. Now that it is finished, make sure that others see the benefits of using simulation.

For the presentation, make sure you know your audience. If the audience is not technical, and they probably won't be, then they will not be interested in how you built the model or how the model works. If you want to spend time explaining the modeling constructs then please schedule a separate technical meeting for those who are interested. The rest of the audience will really appreciate that. The items that need to be in the results presentation include:

- The purpose of the model

- The scope of the model

- The high level process flow

- The assumptions made during the modeling process

- The results by way of answering questions (not just showing numbers)

- The cost/benefit analysis 


\section{Clark, and Krahl}

In the final presentation, answering the questions the model was built to answer along with the cost/benefit analysis is more important than presenting tables of numbers. Have the numbers available in the written up analysis available for those who are interested. When you do provide numbers, be careful not to provide just the averages. Make sure that every time you show the average of a performance measure, you also include a measure of the variance as well.

\section{CONCLUSION}

The most important thing to remember about model building is that it is a process. Good planning, execution, and documentation techniques will help you to become a successful modeler. We hope that you have found this roadmap helpful. If you use our advice it will increase the probability that your first simulation project will be a successful one.

\section{REFERENCES}

Banks, J., J. S. Carson, B. L. Nelson, and D. M. Nicol. 2010. Discrete-Event System Simulation, 5th ed. Upper Saddle River, New Jersey: Prentice-Hall.

Kotiadis, K. and S. Robinson 2008. Conceptual Modelling: Knowledge Acquisition and Model Abstraction. In Proceedings of the 2008 Winter Simulation Conference, ed. S. J. Mason, R. R. Hill, L. Mönch, O. Rose, T. Jefferson, J. W. Fowler. 951-958. Piscataway, New Jersey: Institute of Electrical and Electronics Engineers.

Krahl, D. 2005. Debugging Simulation Models. In Proceedings of the 2005 Winter Simulation Conference, ed. M. E. Kuhl, N. M. Steiger, F. B. Armstrong, and J. A. Joines, 62-68. Piscataway, New Jersey: Institute of Electrical and Electronics Engineers.

Law, A. M. 2007. Simulation Modeling and Analysis, 4th ed. New York: McGraw-Hill.

Musselman, K.J. 1993. Guidelines for Simulation Project Success. In Proceedings of 1993 Winter Simulation Conference, ed. G. W. Evans, M. Mollaghasemi, E. C. Russell, and W. E. Biles, 58-64. Piscataway, New Jersey: Institute of Electrical and Electronics Engineers.

Sargent, R. G. 2009. Verification and Validation of Simulation Models. In Proceedings of the 2009 Winter Simulation Conference, ed. M. D. Rossetti, R. R. Hill, B. Johansson, A. Dunkin, and R. G. Ingalls, 162-176. Piscataway, New Jersey: Institute of Electrical and Electronics Engineers.

\section{AUTHOR BIOGRAPHIES}

DAVID KRAHL is Vice President of Technical Sales with Imagine That Inc. Over the past 20 years he has been involved with all aspects of the simulation industry including training, consulting, support, development, and technical sales. He has worked extensively with a range of simulation programs including ExtendSim, SLAM II, TESS, Factor, AIM, GPSS, SIMAN, XCELL+, and MAP/1. He has taught the System Simulation course at Golden Gate University and consulted in the areas of manufacturing, communications, health care, and transportation for corporate clients. In addition, he has authored or co-authored numerous papers on simulation modeling, including how to debug models, simulation architecture, education, and training, and the ExtendSim modeling environment. Actively involved in the simulation community, he has served as Publicity, and Vendor Track Chair for past Winter Simulation Conferences and is working on the Conference Committee for the Simulation Solutions Conference. He received an MS in Project and Systems Management in 1996 from Golden Gate University and a BS in Industrial Engineering in 1986 from the Rochester Institute of Technology. His email address is DaveK@ExtendSim.com. 
ROBIN CLARK is a co-founder and partner at the Quantitative Modeling \& Training Group (QMT) which offers simulation courses as well as coaching services. At QMT he is a simulation instructor, model builder, and block-level developer. As part of his consulting practice he has designed custom components and built numerous simulation models for clients in the areas of health care, pharmaceutical manufacturing, automotive manufacturing, consumer goods manufacturing, and supply chains. Although he has spent considerable time modeling processes, his primary passion is teaching and mentoring students. He has developed several web-based and face-to-face simulation courses including graphical simulation databases, simulation development environments, rate-based modeling, and simulation modeling for health care. In the last 8 years he has trained over 400 beginner and advanced simulation students to use the ExtendSim simulation software. He has a BS in Physics from Tennessee Technological University and an MS in Management Science from the University of Tennessee. He is a member of the American Society for Training and Development (ASTD), the Society of Manufacturing Engineers (SME), and the Association of Operations Management (APICS). His e-mail addresses is: Clark@QMTGroup.com. 